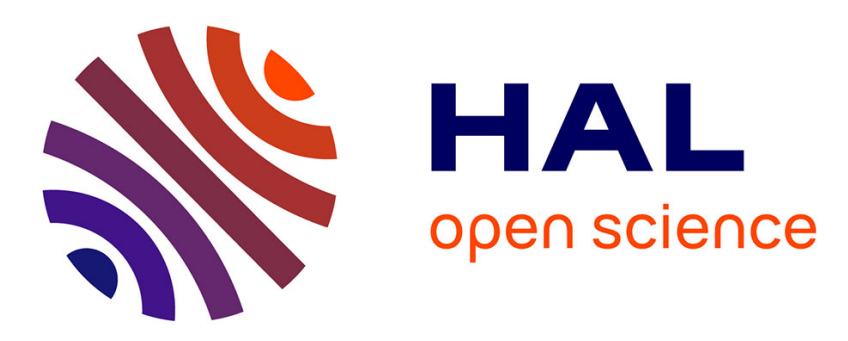

\title{
Sur l'utilisation de la méthode de Kelvin pour l'étude des travaux de sortie des surfaces inhomogènes
}

\author{
L. Soonckindt, J. Bonnet, L. Lassabatère
}

\section{To cite this version:}

L. Soonckindt, J. Bonnet, L. Lassabatère. Sur l'utilisation de la méthode de Kelvin pour l'étude des travaux de sortie des surfaces inhomogènes. Revue de Physique Appliquée, 1979, 14 (8), pp.795-798. 10.1051/rphysap:01979001408079500 . jpa-00244662

\section{HAL Id: jpa-00244662 https://hal.science/jpa-00244662}

Submitted on 1 Jan 1979

HAL is a multi-disciplinary open access archive for the deposit and dissemination of scientific research documents, whether they are published or not. The documents may come from teaching and research institutions in France or abroad, or from public or private research centers.
L'archive ouverte pluridisciplinaire HAL, est destinée au dépôt et à la diffusion de documents scientifiques de niveau recherche, publiés ou non, émanant des établissements d'enseignement et de recherche français ou étrangers, des laboratoires publics ou privés. 


\title{
Sur l'utilisation de la méthode de Kelvin pour l'étude des travaux de sortie des surfaces inhomogènes
}

\author{
L. Soonckindt, J. Bonnet et L. Lassabatère \\ Centre d'Etudes d'Electronique des Solides ( $\left.{ }^{*}\right)$, Université des Sciences et Techniques du Languedoc, USTL, \\ place Eugène-Bataillon, 34060 Montpellier Cedex, France
}

(Reçu le 23 février 1979, réviséle 15 mai 1979, accepté le 15 mai 1979)

\begin{abstract}
Résumé. - Après avoir rappelé de façon générale les problèmes posés, au niveau de la mesure et de son interprétation, pour les inhomogénéités spatiales des surfaces réelles et propres, on s'attache à l'analyse des topographies de travail de sortie effectuées par la méthode de Kelvin. Après avoir précisé l'influence des effets de bord, de la dimension de l'électrode, et montré la nécessité de travailler à résolution constante (donc à distance constante), on donne un exemple d'application correspondant à un cas concret et on rappelle les résultats obtenus par d'autres méthodes.
\end{abstract}

\begin{abstract}
This paper deals with the meaning of work function topographies obtained by the Kelvin method. We first recall the contribution of the fringing field on the edges of the condenser and that of the vibrating electrode dimension to the measured contact potential ; then, we show the necessity of working with a constant resolution, i.e. with a constant electrode-semiconductor-distance. At last we give an experimental example and a background of the results obtained by other methods.
\end{abstract}

1. Introduction. - Le travail de sortie $\boldsymbol{W}_{\varphi}$ est une caractéristique importante des matériaux et plus précisément de leur surface et de l'état cristallin et électronique de celle-ci. Dans le cas des semi-conducteurs il s'exprime au moyen de la relation :

$$
W_{\varphi}=\chi+E_{\mathrm{c}}-E_{\mathrm{F}}-a V_{s}^{Y}
$$

où $\chi$ est l'affinité électronique, $E_{\mathrm{c}}-\dot{E}_{\mathrm{F}}$ la différence d'énergie entre le bord inférieur de la bande de conduction en volume et le niveau de Fermi, $V_{s}$ la hauteur de la barrière en surface.

Les méthodes de mesure du travail de sortie reposent essentiellement sur l'étude de l'émission électronique sous l'action de la lumière (photoémission), de la température (émission thermoélectronique), d'un champ électrique, de l'interaction de la surface avec un pinceau électronique incident (microscope à miroir, microscope à balayage), de la différence de potentiel de contact entre le matériau à étudier et une électrode de référence (Kelvin, Zisman [1], [2]). Si les techniques actuelles permettent d'interpréter correctement les résultats obtenus sur des surfaces homogènes, il n'en va pas de même si la surface est hétérogène. Dans le

(*) Associé au C.N.R.S. cas d'un semiconducteur en particulier, du fait de la répartition non homogène des défauts cristallins, des atomes adsorbés, etc..., la barrière de surface et parfois l'affinité électronique varient de façon notable d'un point à un autre de l'échantillon [3]. Des inhomogénéités de volume peuvent aussi entraîner des variations locales de la position du niveau de Fermi qui se traduiront par des variations du travail de sortie. La surface est ainsi divisée en domaines dont les dimensions peuvent être aussi faibles que quelques dizaines d'angströms. Ces domaines peuvent jouer un rôle fondamental :

- dans les études de photoémission : en effet c'est à partir des zones de plus faible travail de sortie que commencera l'émission électronique ;

- dans les études de mesure du potentiel de contact : la valeur mesurée est en fait une valeur moyennée sur toute la surface située en regard de l'électrode. Ceci peut expliquer la divergence entre certains résultats expérimentaux publiés ces dernières années et concernant en particulier des surfaces clivées de semiconducteurs.

Il s'avère donc indispensable de préciser la signification de la mesure qui est faite. C'est l'objet de cette note consacrée aux relevés de topographies du travail de sortie par la méthode de Kelvin et aux problèmes qu'ils entraînent. 
2. Technique du condensateur vibrant de Kelvin. La technique du condensateur vibrant de KelvinZisman consiste à mesurer la différence des travaux de sortie $W_{\varphi s c}$ et $W_{\varphi \mathrm{M}}$ entre la surface à étudier et une électrode de référence. Pour cela, on constitue avec ces deux matériaux un condensateur dont on fait varier périodiquement la distance inter-électrodes. S'il existe une différence de potentiel de contact $V_{\mathrm{cp}}$ entre les armatures, la vibration induit une différence de potentiel alternative qu'on cherche à annuler au moyen d'un générateur de force électromotrice $E$ placé en opposition avec $V_{\text {cp }}$. A l'équilibre :

$$
V_{\mathrm{cp}}+E=0
$$

avec

$$
V_{\mathrm{cp}}=\frac{W_{\varphi \mathrm{sc}}-W_{\varphi \mathrm{M}}}{q} .
$$

Pour un élément $\mathrm{j}$ de surface homogène d'aire $S_{\mathrm{j}}$ le courant mesuré $i_{\mathrm{j}}$ s'écrit (4) :

$$
i_{\mathrm{j}}=\left(V_{\mathrm{cpj}}+E\right) \frac{\varepsilon S_{\mathrm{j}}}{e^{2}} \frac{\mathrm{d} e}{\mathrm{~d} t}
$$

où $e$ est la distance inter-électrodes, $\varepsilon$ la constante du diélectrique. Si la surface considérée est composée de deux surfaces homogènes $S_{1}$ et $S_{2}$, de travaux de sortie différents $\left(S=S_{1}+S_{2} \equiv\right.$ surface de la contreélectrode) on mesurera un courant total $i_{\mathrm{T}}=i_{1}+i_{2}$ qui s'annulera pour

$$
-E=\frac{V_{\mathrm{cp} 1} S_{1}+V_{\mathrm{cp} 2} S_{2}}{S_{1}+S_{2}}
$$

$-E=\overline{V_{c p}}$ donnera la valeur du potentiel de contact moyen pour l'ensemble des zones mesurées.

En posant :

$$
f_{\mathrm{j}}=\frac{S_{\mathrm{j}}}{\sum S_{\mathrm{j}}}
$$

on peut généraliser la relation (5) soit :

$$
\overline{V_{\mathrm{cp}}}=\sum f_{\mathrm{j}} V_{\mathrm{cpj}}
$$

ce qui s'écrit en passant aux travaux de sortie

$$
\overline{W_{\varphi}}=\sum f_{j} W_{\varphi \mathbf{j}}
$$

où $\overline{W_{\varphi}}$ est le travail de sortie moyen de la zone située face à l'électrode et $W_{\varphi \mathrm{j}}$ le travail de sortie de l'élément de surface $S_{\mathrm{j}}$. On retrouve là une relation déjà employée par Herring et Nichols [5] pour les surfaces polycristallines.

3. Effets de la réduction de la dimension de la contre-électrode sur la mesure. - 3.1 EFFETS DE BORD. - Lorsqu'on diminue la dimension de la contreélectrode pour pouvoir étudier la topographie d'une surface, on se heurte rapidement au problème des

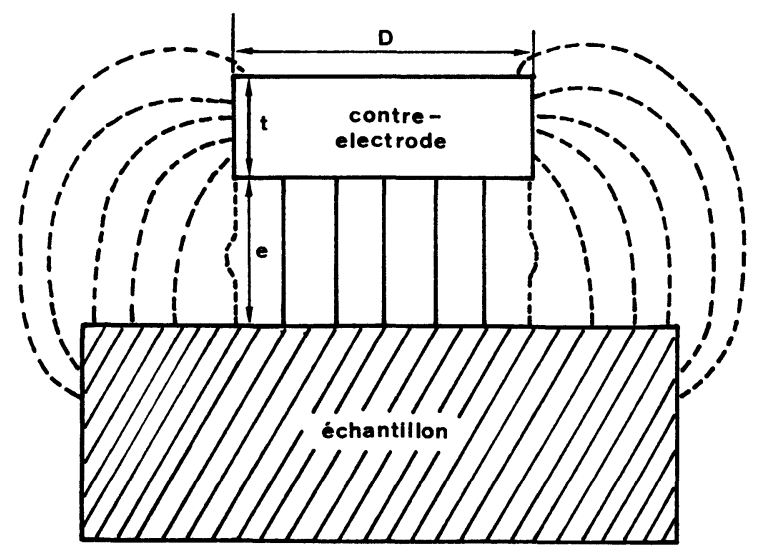

Fig. 1. - Lignes de champ électrique entre la contre-électrode et l'échantillon. En traits pleins les lignes de champ utiles. En tirets les lignes de champs parasites.

[Field flux of the electrode-semiconductor condenser Full lines : the "useful " field flux Dashed lines : the spurious field flux.]

effets de bord. Celui-ci naît du fait qu'à la capacité utile formée par la contre-électrode et la portion de surface de l'échantillon située en regard va s'ajouter une capacité parasite (voir Fig. 1) qui dépend des caractéristiques géométriques de la contre-électrode, de la surface du matériau et de son état structural.

Dans le cas d'un condensateur formé par une surface infinie et une surface finie, toutes deux homogènes, les capacités utile et parasite $C_{\mathrm{u}}$ et $C_{\mathrm{p}}$ s'expriment respectivement [6] avec les notations de la (Fig. 1) par :

$$
\begin{aligned}
C_{\mathrm{u}}=\frac{1,113 D^{2}}{16 e} \mu \mu \mathrm{F} \\
\begin{aligned}
C_{\mathrm{p}}=\frac{1,113 D}{8 \Pi} & \left(\operatorname{Ln} \frac{8 \Pi D}{e}-3+\left(1+\frac{t}{e}\right) \times\right. \\
& \left.\times \operatorname{Ln}\left(1+\frac{t}{e}\right)-\frac{t}{e} \operatorname{Ln} \frac{t}{e}\right) \mu \mu \mathrm{F} .
\end{aligned}
\end{aligned}
$$

3.2 INFLUENCE DE LA DISTANCE SUR LA MESURE. Les relations précédentes montrent que l'effet parasite est d'autant plus important que le diamètre $D$ de la contre-électrode est réduit et que la distance interélectrodes $e$ est grande. En effet lorsque la surface est hétérogène, la valeur de $E$ correspondant à l'équilibre est modifiée au fur et à mesure que $e$ croît du fait que $C_{\mathrm{p}}$ englobe des zones plus étendues de la surface. Ceci se traduit par une modification de la valeur moyenne du potentiel de contact.

On peut pallier à ceci de deux façons. Tout d'abord, il est possible de munir la contre-électrode d'une garde : Danyluck [7] a montré qu'on réduisait alors les effets parasites dus à la variation de distance. L'inconvénient de cette méthode est qu'elle se traduit par une augmentation de la surface de la contre-électrode et que la garde risque d'être une gêne si la surface à étudier présente de fortes dénivellations.

La deuxième solution consiste à maintenir constante la distance $e$. Pour cela, on utilise un dispositif d'asservissement (4) de la distance inter-électrodes 
qui permet de travailler à résolution constante sur une même surface, et de conserver cette résolution pour les différents échantillons. Ainsi les mesures comparatives effectuées sur une série de surfaces au cours d'un même cycle d'étude prennent-elles toute leur signification.

4. Simulation de mesure de potentiel de contact sur des surfaces hétérogènes. - A partir de la relation (5) démontrée précédemment, nous avons simulé des mesures de potetiel de contact sur une surface hétérogène constituée par une zone homogène de potentiel $V_{\mathrm{cp} 1}=0,6 \mathrm{~V}$ au milieu de laquelle se trouve incluse une zone homogène de potentiel $V_{\mathrm{cp} 2}=0,1 \mathrm{~V}$, dont la largeur $l_{0}$ est inférieure ou égale au diamètre de la contre-électrode. Ceci correspond à un cas concret, par exemple à une zone parfaitement structurée sans états occupés (cas de GaSb bien clivé) dans laquelle on trouve un domaine avec des marches, des atomes relaxés, etc...

On peut préciser alors la signification des mesures et déterminer la variation de charge superficielle détectable dans le cas d'un échantillon de $\mathrm{GaSb}$, de type $\mathrm{n}$ par exemple $\left(N_{\mathrm{D}}=10^{18} \mathrm{~cm}^{-3}\right)$. Supposons une zone homogène parfaitement clivée avec des bandes plates $\left(V_{\mathrm{s} 2}=0\right)$ et une zone hétérogène avec une densité de charges en surface

$$
N_{\mathrm{s}}=3,1 \times 10^{12} \mathrm{~cm}^{-2}
$$

qui donne une zone de déplétion en surface et une courbure de bande :

$$
\left|V_{\mathrm{s} 1}\right|=\frac{q N_{\mathrm{s}}^{2}}{2 \varepsilon N_{\mathrm{D}}}=0,6 \mathrm{~V} \text {. }
$$

Si la sensibilité de la mesure est de $5 \times 10^{-3} \mathrm{~V}$ et si la surface de l'électrode est $S_{1}+S_{2}=3 \mathrm{~mm}^{2}$ cela signifie qu'on pourra détecter une variation de charge en passant de $S_{1}$ à $S_{2}$ si :

$$
S_{1}>\frac{5 \times 10^{-3}}{6 \times 10^{-1}}\left(S_{1}+S_{2}\right)=0,025 \mathrm{~mm}^{2} \text {. }
$$

A cette surface $S_{1}$ il correspondra une charge globale égale à $7,75 \times 10^{8} q$. Si cette même charge était répartie sur des marches de $2 \mathrm{~mm}$ de long, à raison d'une charge élémentaire par atome, cela correspondrait à un total de 240 marches environ soit une marche tous les $520 \AA$ en moyenne.

(Fig. 2) sont représentées les variations théoriques de $E$ déduites de la relation (5) pour des zones perturbées larges de 0,$1 ; 0,2 ; 0,5 ; 1$ et $2 \mathrm{~mm}$. Cette dernière dimension correspond au diamètre de la contre-électrode.

De l'observation de la famille de courbes de la figure 2 on peut tirer quelques conclusions :

- pour une dimension de contre-électrode donnée, plus la largeur de la zone perturbée sera faible, plus l'effet sur $|E|$, donc sur $V_{\mathrm{cp}}$, sera faible. Une étude
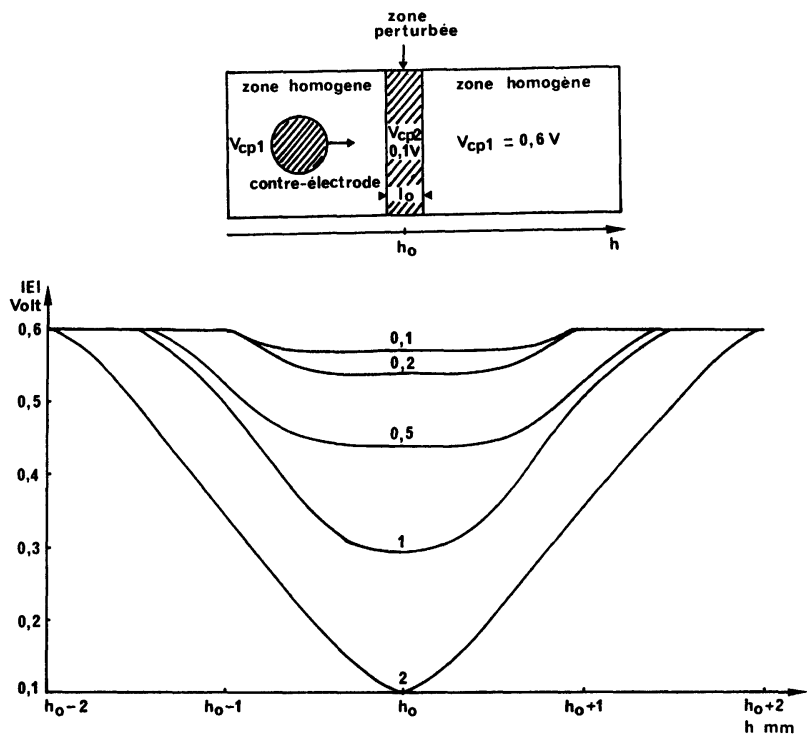

Fig. 2. - Variation de la contre-tension $|E|$ dans une région perturbée. En paramètre la largeur en millimètres de la zone perturbée. $h_{0}$ est l'abscisse du centre de la zone perturbée.

[Variation of the opposite tension $|E|$ in a perturbated area. The parameter is the width of the perturbated area in millimeters $h_{0}$ is the absciss of the perturbated area center.]

plus fine implique une réduction du diamètre de l'électrode mais on augmente du même coup les effets parasites. On peut le faire cependant et dèscendre jusqu'à $100 \mu \mathrm{m}$ [8] dans le cas des diélectriques car la d.d.p. de contact à mesurer est alors très importante ;

- avec une contre-électrode plane on ne pourra mesurer la valeur vraie du potentiel de contact d'une zone homogène que si sa largeur est supérieure ou égale à celle du diamètre de la contre-électrode. A titre d'application, nous donnons (Fig. 3) la variation du travail de sortie $\Delta W_{\varphi}$ d'un échantillon de silicium sur la moitié duquel on a déposé une couche de $2500 \AA$ de palladium.

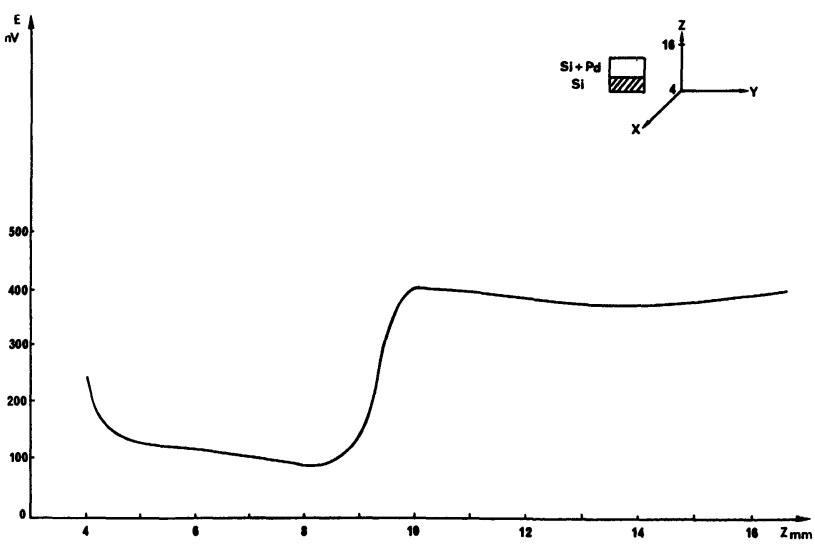

Fig. 3. - Variation de la contre-tension $E$ mesurée sur une plaquette de silicium à demi recouverte de palladium.

[Variation of the opposite tension $E$ measured on a slice of silicium half-covered with palladium.] 
5. Application. $-E$ a été enregistrée continûment avec une sonde de $1 \mathrm{~mm}$ de diamètre environ: On peut noter que la zone de raccordement est "du même ordre de grandeur.

6. Topographies réalisées par d'autres méthodes. Il existe peu d'études topographiques des surfaces semiconductrices et exception faite des travaux de Van Laar et al. [9] peu de résultats ont été publiés dans ce domaine.

D'autres méthodes ont été utilisées pour réaliser des topographies du travail de sortie de surfaces semiconductrices ou métalliques mais ne paraissent pas avoir donné lieu à des études systématiques. C'est ainsi qu'en photoémission Guichar [10] a observé des variations du travail de sortie en différents points d'une même surface de $\mathrm{GaSb} n$ fraîchement clivée. Avec une source à hélium la résolution spatiale est de $1,5 \times 0,5 \mathrm{~mm}^{2}$ (avec une précision de $10 \mathrm{meV}$ ), donc tout à fait comparable à celle obtenue avec la tech- nique de Kelvin. On pourrait certainement améliorer ces résultats en utilisant le rayonnement d'un synchrotron mais ceci n'a pas été fait à notre connaissance.

A partir de l'interaction d'un faisceau d'électrons de faible énergie $(1,5 \mathrm{eV})$ avec une surface on peut, avec la technique du microscope à miroir [11] obtenir une résolution de l'ordre de $100 \mu \mathrm{m}^{2}$ avec une précision de $10 \mathrm{mV}$.

7. Conclusion. - En conclusion, on peut donc dire que si des techniques utilisant des faisceaux électroniques ou lumineux permettent d'atteindre des résolutions supérieures à la méthode de Kelvin, elles présentent l'inconvénient majeur en contrepartie de pouvoir perturber la surface. De ce fait l'analyse des topographies obtenues par la technique du condensateur vibrant conserve tout son intérêt, même si les dimensions des zones analysables restent de l'ordre du millimètre.

\section{Bibliographie}

[1] Kelvin, Philos. Mag. 5 (1898) 82.

[2] Zisman, W. A., Rev. Sci. Instrum. 3 (1932) 367.

[3] Viljoen, P. E., Jazzar, M. S., Fischer, T. E., Surf. Sci. 32 (1972) 506.

[4] Bonnet, J., Soonckindt, L., Lassabatère, L., Congrès MECO'77, Zurich (1977) 16

[5] Herring, C., Nichols, M. H., Rev. Mod. Phys. 21 (1949) 185.

[6] Curtis, H. L., Scott, A. H., ASTM Proc. 36 Pt II (1936) 815.
[7] Danyluk, S., J. Phys. E 5 (1972) 478.

[8] SHOCKLey, W., HoOPer, W. W., Queisser, H. J., SChroen, W., Surf. Sci. 2 (1964) 277.

[9] Van LaAR, J., Huisser, A., Van Rooy, T. L., J. Vac. Sci. Technol. 14 (1977) 894.

[10] Guichar, G. M., Thèse (1978) Paris.

[11] Dupuy, J. C., Laydevant, L., Etienne, S., J. Phys. E 9 (1976) 176 